\title{
Untersuchungen zur Interpretation des Trijodthyronintests
}

\author{
Von G. Meuret und G. HoffmanN \\ Aus der Nulelearmedizinischen Abteilung (Leiter: Prof. Dr. G. Hoffmann) der Med. Univ.-Klinik (Komm. Direktor: \\ Prof. Dr. J. Schirmeister) Freiburg i. Br.
}

(Eingegangen am 17. Juli 1968)

Die Untersuchung der Grundlagen des zur Diagnostik von Schilddrüsenerkrankungen dienenden Trijodthyronintests ergab folgende Interpretation der Testergebnisse:

Mit dem Trijodthyronintest wird in der Regel indirekt das Anlagerungsvermögen für Schilddrüsenhormone von Plasmaproteinen spezifisch hoher Affinität bestimmt. Meist ist der Einfluß endogener Schilddrüsenhormone für das Ergebnis irrelevant. Ubersteigt jedoch der Spiegel das spezifische Transportvermögen des Plasmas, so wird der Test von Proteinen niedriger Affinität stärker mitbestimmt. Auch können pathologische Plasmafaktoren, wie z. B. vermehrte Lipide, den Testparameter verändern.

\section{Studies on the interpretation of the triiodotbyronine test}

The triiodothyronine test measures indirectly the binding capacity of plasma proteins with high affinities for thyroid hormones. Usually the results are not affected by endogenous thyroid hormones. However, if the level of the thyroid hormones exceeds this binding capacity, proteins with lower affinities will influence the results. Also pathological compositions of the plasma, like an increased lipid level, may alter the triiodothyronine test.

Der 1957 von Hamolsky und Mitarbeitern (1) eingeführte Trijodthyronintest wird heute häufig zur Diagnostik von Schilddrüsenerkrankungen mit herangezogen. Bei der zuerst verwendeten Methodik (1) wurde dem Blut in vitro ${ }^{131} \mathrm{~J}$-Trijodthyronin zugesetzt und nach Bebrütung der an den gewaschenen Erythrocyten verbliebene Radioaktivitätsanteil bestimmt. In neuerer Zeit wurden anstatt der Erythrocyten auch Ionenaustauscher $(2,3)$ oder Sephadex (4) verwendet.

Die vorliegenden Untersuchungen sollen zur Klärung der Frage nach der Bedeutung des gemessenen Testparameters beitragen.

\section{Methodik}

Mit Heparin versetzte Blutproben $(20-30 \mathrm{E} / \mathrm{m} /$ Blut) wurden auf einen Hämatokritwert von 40 eingestellt.

Zur Bestimmung der Trijodthyroninaufnahme der Erythrocyten $\left.\left(\%{ }^{131} \mathrm{~J}-\mathrm{T}_{3} \text {-E.A. }\right)^{1}\right)$ wurden jeweils $2,5 \mathrm{ml}$ des präparierten Bluts mit biṣ zu $0,04 \mu \mathrm{g}{ }^{131} \mathrm{~J}$-Trijodthyronin $\left({ }^{131} \mathrm{~J}-\mathrm{T}_{3}\right)$ versetzt, die Radioaktivität im Szintillationszähler gemessen und die vẹschlossenen Proben 2 Stdn. bei $37^{\circ}$ im Wasserbad unter Schütteln inkubiert. Anschließend wurden die Erythrocyten $5 \mathrm{mal} \mathrm{mit} \mathrm{je}$ $10 \mathrm{ml}$ 0,9proz. $\mathrm{NaCl}-\mathrm{L}$ ösung innerhalb von $30-40 \mathrm{Min}$. gewaschen, erneut die Radioaktivität bestimmt und der prozentuale Anteil der an den Erythrocyten verbliebenen Radioaktivität, bezogen auf den Ausgangswert, berechnet.

Den angegebenen Meßwerten liegen 3-7fach Bestimmungen zugrunde. Die methodische Fehlerbreite des Mittelwerts einer Dreifachbestimmung lag bei $\pm 3,3 \%\left(\sigma_{\dot{x}}\right)$.

${ }^{127} \mathrm{~J}-\mathrm{L}=\mathrm{T}$ rijodthyronin $\left({ }^{127} \mathrm{~J}-\mathrm{T}_{3}\right)$ oder ${ }^{127} \mathrm{~J}-\mathrm{L}-\mathrm{Thyroxin} \quad\left({ }^{127} \mathrm{~J}-\mathrm{T}_{4}\right)$ wurden dem Blut in $0,02 \mathrm{~N} \mathrm{NaOH}$ gelöst zugesetzt (pro $\mathrm{ml}$ Blut $0,01 \mathrm{ml}$ der Lösung). Zur Verdünnung des Plasmas wurde ein $\mathrm{NaCl}$-Borat-Puffer mit $\mathrm{pH}=7,4$ verwendet.

\section{Ergebnisse}

Die Frage nach der Art der Trijodthyroninanlagerung an Erythrocyten im Plasma wurde mit folgender Versuchsanordnung untersucht: Blut, mit ${ }^{131} \mathrm{~J}-\mathrm{T}_{3}$ als Tracer, wurde zusammen mit verschiedenen Mengen

1) Ableürzungen: ${ }^{131} \mathrm{~J}-\mathrm{T}_{3}$-E.A. $={ }^{131} \mathrm{~J}$-Trijodthyronin-Aufnahme der Erythrocyten; HK = Hämatokritwert; TBG = Thyroxin bindendes Globuling.
${ }^{127} \mathrm{~J}-\mathrm{T}_{3}$ versetzt und inkubiert. Durch Messung der im Plasma verbliebenen $\mathrm{T}_{3}$-Menge wurde auch der von den ungewaschenen Erythrocyten aufgenommene Anteil des zugesetzten $T_{3}$ bestimmt.

Repräsentativ für die an 4 Normalpersonen und 2 Graviden durchgeführten Untersuchungen sind die in Tabelle 1 aufgeführten Meßergebnisse. - Die $T_{3^{-}}$

Tab. 1

Trijodthyroninanlagerung an ungewaschene und gewaschene Erythrocyten

\begin{tabular}{|c|c|c|c|c|}
\hline $\begin{array}{c}\mu \mathrm{g} / 100 \mathrm{ml} \\
\text { zugegebenes } \mathrm{T}_{3} \\
\mathrm{im} \text { Plasma }\end{array}$ & \multicolumn{2}{|c|}{$\begin{array}{c}\text { Euthyreose } \\
(\mathrm{n}=4) \\
\%{ }^{131} \mathrm{~J}-\mathrm{T}_{3}-\mathrm{E} . A . \\
\text { unge- } \\
\text { waschene waschene } \\
\text { Erythrocyten }\end{array}$} & \multicolumn{2}{|c|}{$\begin{array}{c}\text { Gravidität } \\
(n=2) \\
\%{ }^{131} \mathrm{~J}-\mathrm{T}_{3}-\mathrm{E} . \mathrm{A} . \\
\text { unge- } \\
\text { waschene waschene } \\
\text { Erythrocyten }\end{array}$} \\
\hline $\begin{array}{r}0,3 \\
2 \\
5 \\
10 \\
20 \\
50 \\
100 \\
200 \\
500 \\
1000 \\
4000 \\
10000 \\
20000\end{array}$ & $\begin{array}{l}\overline{17,9} \\
18,9 \\
19,3 \\
20,0 \\
21,4 \\
22,9 \\
25,0 \\
25,6 \\
27,2 \\
-\end{array}$ & $\begin{array}{r}\overline{5,6} \\
5,9 \\
6,3 \\
7,9 \\
8,9 \\
10,6 \\
11,0 \\
12,2 \\
12,0 \\
= \\
-\end{array}$ & $\begin{array}{l}17,0 \\
19,4 \\
19,0 \\
21,0 \\
21,0 \\
23,0 \\
24,0 \\
25,0 \\
28,0 \\
26,0 \\
27,0 \\
27,0 \\
31,0\end{array}$ & $\begin{array}{r}3,5 \\
3,6 \\
3,7 \\
4,7 \\
5,3 \\
5,4 \\
6,0 \\
9,7 \\
9,7 \\
9,9 \\
11,9 \\
12,6 \\
12,9\end{array}$ \\
\hline
\end{tabular}

Aufnahme der ungewaschenen Erythrocyten beider Gruppen war etwa gleich groß. Sie lag bei niedrigen $\mathrm{T}_{\mathbf{3}}$-Konzentrationen um $17-20 \%$ der zugesetzten $\mathrm{T}_{3}$-Menge. Der entsprechende, an den gewaschenen Erythrocyten gemessene $T_{3}$-Anteil, machte dagegen nur etwa $1 / 4$ hiervon aus. Der abwaschbare $T_{3}$-Anteil war im Blut von Graviden wesentlich größer als bei euthyreoten Probanden.

Mit ansteigender $T_{3}$-Konzentration nahm bei beiden Gruppen die $T_{3}$-Anlagerung an die ungewaschenen Erythrocyten in gleicher Weise kontinuierlich zu. Bei gewaschenen Erythrocyten war der ebenfalls kontinuierliche $\mathrm{T}_{3}$-Anstieg durch ein stufenartiges Anwachsen unterbrochen (Abb. 1). Diese Stufen fanden sich bei Normalpersonen im Bereich zwischen $10-20 \mu \mathrm{g} /$ $100 \mathrm{ml}$ exogenem $\mathrm{T}_{3}$; bei Graviden zwischen $10-20 \mu \mathrm{g} /$ $100 \mathrm{~m} l$ und $1000-4000 \mu \mathrm{g} / 100 \mathrm{~m} l$ exogenem $\mathrm{T}_{3}$. 


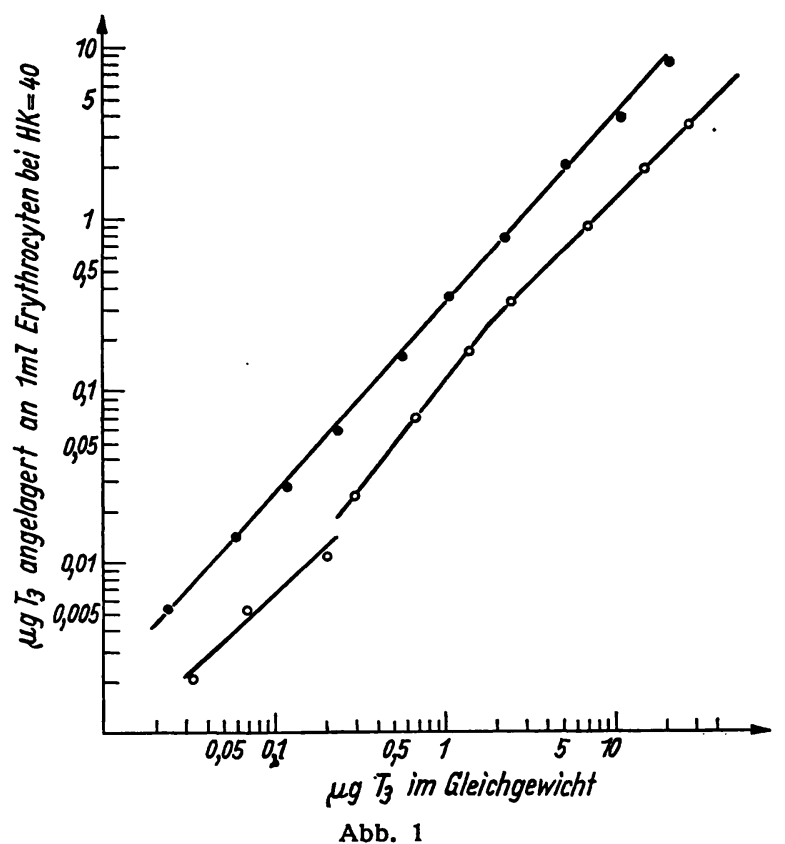

$101 \mathrm{~J}$ Trijodthyroninanlagerung an ungewaschene ( $\left.-^{-}\right)$und gewaschene (o-o) Erythrocyten in Abhängigkeit vom $\mathrm{T}_{\mathrm{s}}$-Spiegel im
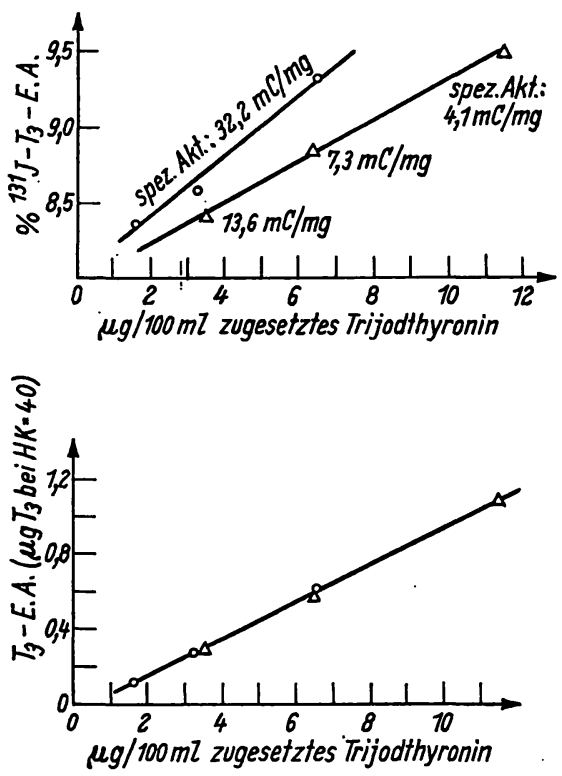

Abb. 2

Prozentuale ${ }^{131} \mathrm{~J}-\mathrm{T}_{3}-$ E.A. und absolute $T_{3}$-E.A. in Abhängigkeit vom $\mathrm{T}_{3}$-Spiegel im Plasma

In Abbildung 1 wurden die Logarithmen der aus diesen Meßwerten berechneten Trijodthyroninaufnahme (in $\mu \mathrm{g})$ von ungewaschenen und gewaschenen Erythrocyten zu der im Gleichgewicht stehenden $T_{3}$-Konzentration (in $\mu \mathrm{g}$ ) in Beziehung gesetzt (Mittelwerte von 4 Normalpersonen). Es ergeben sich Geraden, welche der von FreundLICH (5) formulierten Gleichung für Adsorptionsvorgänge $\left(\lg \frac{x}{m}=\frac{1}{p} \cdot \operatorname{lgc}+\lg \beta\right)$ gehorchten. Während jedoch für die $T_{3}$-Anlagerung an ungewaschene Erythrocyten eine einheitliche Funktion vorlag, wies die Kurve der $\mathrm{T}_{3}$-Anlagerung an gewaschene eine Stufe im Bereich zwischen $10-20 \mu \mathrm{g} \mathrm{T}$ auf und hatte $z$ wischen $50-100 \mu \mathrm{g} / 100 \mathrm{~m} l$ größere Steigerung als bei den übrigen untersuchten Konzentrationen.

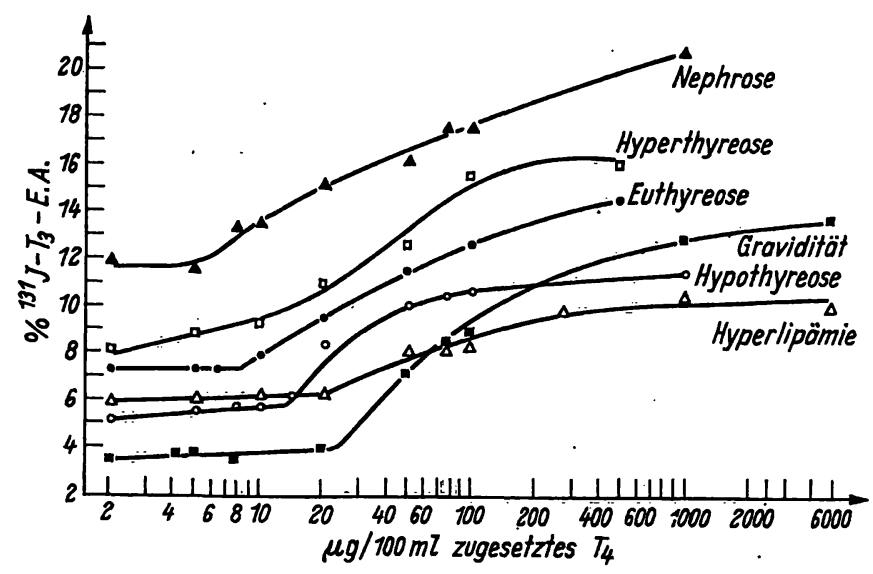

Abb. 3

Prozentuale ${ }^{131} \mathrm{~J}-\mathrm{T}_{3}-$ E.A. nach Zugabe von ${ }^{127} \mathrm{~J}-\mathrm{T}_{4}$ zum Blut

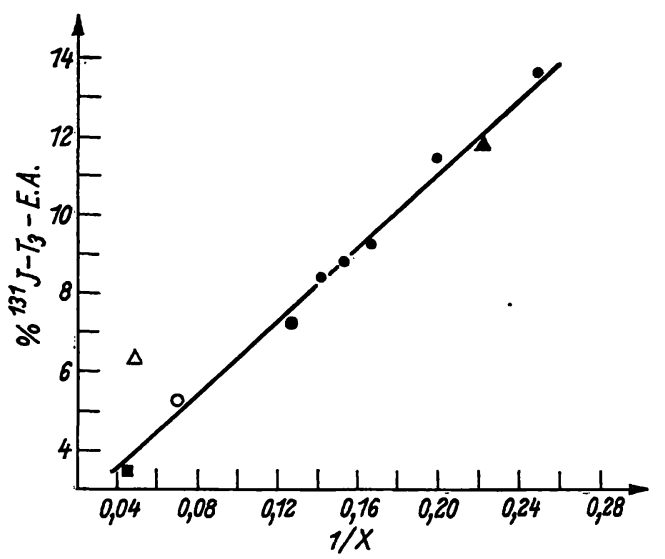

Abb. 4

Prozentuale ${ }^{181} \mathrm{~J}-\mathrm{T}_{3}-\mathrm{E} . \mathrm{A}$. in Beziehung zu dem nicht von endogenen Schilddrüsenhormonen besetzten Bindungsvermögen von Plasmaproteinen mit spezifisch hoher Affinität (,,spezifisches Transportvermögen" des Plasmas) Euthyreose, Gravidität, Euthyreose nach Plasmaverdünnung
O Hypothyreose, $\Delta$ Nephrose, $\triangle$ Hyperlipämie
$\dot{x}=$ nicht von endogenen Schilddrüsenhormonen besetztes , spezifisches $\mathbf{x}=$ nicht von endogenen Schilddrüsenhormonen besetztes , spezifisches

Die Abhängigkeit der $T_{3}$-Aufnahme an Erythrocyten vom Spiegel der Schilddrüsenhormone im Plasma wurde durch Zusatz von ${ }^{127} \mathrm{~J}-\mathrm{T}_{3}$ und ${ }^{127} \mathrm{~J}-\mathrm{T}_{4}$ zum Ansatz untersucht.

${ }^{127} \mathrm{~J}-\mathrm{T}_{3}$ bewirkte eine der Plasmakonzentration proportionale Vermehrung der prozentualen ${ }^{131} \mathrm{~J}-\mathrm{T}_{3}$-E.A. und der absoluten $T_{3}$-E.A., wobei der Zuwachs des ersten Parameters wesentlich niedriger lag als der des zweiten (Abb. 2).

Der Abfall der spezifischen $\mathrm{T}_{3}$-Aktivität (durch Zugabe von ${ }^{127} \mathrm{~J}-\mathrm{T}_{3}$ zum Ansatz) bei diesem Versuch, verursachte eine geringe Erniedrigung der Testwerte (Abb. 2).

Ein ähnliches Ansteigen der prozentualen ${ }^{131} \mathrm{~J}-\mathrm{T}_{3}$-E.A. wurde zunächst auch nach Zugabe von ${ }^{127} \mathrm{~J}-\mathrm{T}_{4}$ zum Ansatz beobachtet (Abb. 3). Wurde jedoch eine bestimmte Thyroxinmenge überschritten, so stiegen die Kurven unvermittelt steiler an, verliefen dann wieder flacher, um schließlich bei hohen Konzentrationen in ein Plateau überzugehen. Bei zwei untersuchten Hyperthyreosen fehlte der erste, flach verlaufende Kurvenabschnitt.

In Abbildung 4 wurden die Reziprokwerte der zugesetzten $\mathrm{T}_{\mathbf{4}}$-Konzentrationen an der knickartigen Uber- 
gangsstelle der Kurvenabschnitte zu der bei $2 \mu \mathrm{g} / 100 \mathrm{~m} l$ zugesetzten ${ }^{127} \mathrm{~J}-\mathrm{T}_{4}$ gemessenen prozentualen ${ }^{131} \mathrm{~J}-\mathrm{T}_{3}$ E.A. in Beziehung gesetzt. Diese Größen verhielten sich proportional. Auch Werte, die nach Plasmaverdünnung von Normalblut gefunden wurden, gehorchten der Funktion. Andere Verhältnisse lagen offensichtlich bei einem Patienten mit einer essentiellen Hyperlipämie (Gesamtlipide $7682 \mathrm{mg} / 100 \mathrm{ml}$ ) vor.

\section{Diskussion}

Erythrocyten weisen bei der Trijodthyroninanlagerung keine spezifischen Eigenschaften auf, sondern verhalten sich wie ein unspezifisches Adsorbens $(6,7)$. Individuelle oder durch Krankheiten bedingte Veränderungen der Eigenschaften als Adsorbens für $\mathrm{T}_{3}$ scheinen in der Regel nicht vorzuliegen (1). Daher gilt die aus den vorliegenden Untersuchungen mit Erythrocyten abgeleitete Interpretation des Trijodthyronintestes auch für Testmethoden, bei denen andere Adsorbentien, wie z. B. Ionenaustauscher (2, 3 u. a.) oder Sephadex (4) verwendet werden. Unberücksichtigt allerdings bleibt die eventuell unterschiedliche Proteinanlagerung an die verschiedenen Adsorbentien, die - wie unten erörtert werden soll - Einfluß auf das Ergebnis nehmen könnte.

Als Adsorptionsvorgang hat sich auch die Bindung der Schilddrüsenhormone an Plasmaproteine erwiesen, da diese sich, gemäß ihrer Affinität, von den Plasmafraktionen, denen sie angelagert sind, ablösen lassen (8-10).

Das dem Blut beim Trijodthyronintest zugesetzte ${ }^{131} \mathrm{~J}-\mathrm{T}_{3}$ verteilt sich während der Inkubation demnach zwischen den Oberflächen der Plasmaproteine und der Oberfläche des zugesetzten Adsorbens. Es kann angenommen werden, daß das Verteilungsgleichgewicht im wesentlichen vom Größenverhältnis der BindungsAffinitäten und -Kapazitäten der Oberflächen beider Adsorbentien bestimmt wird.

Für das zugesetzte Adsorbens sind Bindungs-Affinität und -Kapazität durch die Testmethodik definiert (durch den Hämatokrit bei der Methode von Hamolsky (1)) oder konstant gehalten (durch Einwiegen des Ionenaustauschers).

Das Testergebnis wird somit von den im Plasma vorliegenden Bindungsmöglichkeiten für $T_{3}$ bestimmt.

Bekanntlich ist hier der Hauptteil der Schilddrüsenhormone einer Proteinfraktion mit spezifisch hoher Affinität angelagert, dem sog. Thyroxin-bindenden Globulin (TBG) (11-13), dessen Affinität zu Thyroxin jedoch etwa 3 mal höher ist, als zu Trijodthyronin $(8-10,14)$. Die Transportkapazität soll, elektrophoretischen Untersuchungen zufolge, normalerweise um $20 \mu \mathrm{g} / 100 \mathrm{~m} / \mathrm{T}_{4}$ betragen '(10). Sie ist bei Hypothyreosen $(15)$ und der Gravidität $(16,17)$ vermehrt und bei der Nephrose verringert $(15,18)$ und soll bei Hyperthyreosen normal sein (10). Auch das thyroxinbindende Praealbumin (TBPA) und Albumin besitzen - eine relativ hohe Thyroxin-Affinität (8).
Der Einfluß der Anlagerunsverhältnisse für Schilddrüsenhormone im Plasma wurde durch in vitro Zugabe von ${ }^{127} \mathrm{~J}-\mathrm{T}_{3}$ bzw. ${ }^{127} \mathrm{~J}-\mathrm{T}_{4}$ analysiert. Úberraschend gering veränderte sich der Testparameter bei Zugabe von Schilddrüsenhormonen, selbst beim Úberschreiten physiologischer Konzentrationen (Abb. 2, 3). Diese Tatsache bestätigen auch in vivo Untersuchungen, bei denen Gesunden Schilddrüsenhormone appliziert wurden $(1,7,19)$. Obwohl zum Teil hyperthyreote Symptome auftraten, änderten sich die Testwerte nicht signifikant.

Wir nehmen an, daß das dem Plasma zugesetzte Thyroxin in niedrigerer Konzentration aufgrund seiner höheren Affinität zu TBG ${ }^{131} \mathrm{~J}_{-} \mathrm{T}_{3}$ aus dieser Fraktion verdrängte. ${ }^{131} \mathrm{~J}-\mathrm{T}_{3}$. lagerte sich dann anderen Plasmafraktionen an, die zwar eine niedrigere Affinität, wahrscheinlich aber eine große Bindungskapazität aufwiesen, so $\mathrm{da} \beta$ die prozentuale ${ }^{131} \mathrm{~J}-\mathrm{T}_{3}$-E.A. nur wenig anstieg. Die Absättigung der Fraktionen spezifisch hoher $\mathrm{T}_{4}$-Affinität, bedeutete deren Ausfall als Anlagerungsmöglichkeit für $T_{4}$. Bei weiterer Erhöhung des $T_{4}$-Spiegels nahm folglich das $T_{4}$-Angebot an die restlichen Fraktionen plötzlich stärker $\mathrm{zu},{ }^{131} \mathrm{~J}-\mathrm{T}_{3}$ wurde in gleichem Maße dort verdrängt, die prozentuale ${ }^{131} \mathrm{~J}-\mathrm{T}_{3}$-E.A. stieg steiler an (Abb. 3).

Die zur Absättigung notwendige $\mathrm{T}_{4}$-Menge (abgelesen an der knickartigen Úbergangsstelle der Kurvenabschnitte in Abb. 3) entspricht somit dem nicht von endogenen Schilddrüsenhormonen besetzten ,spezifischen $\mathrm{T}_{\mathbf{4}}$-Anlagerungsvermögen " des Plasmas (s. auch WALFISH (20)).

Die Reziprokwerte des zur Absättigung erforderlichen $\mathrm{T}_{4}$ waren der prozentualen ${ }^{131} \mathrm{~J}-\mathrm{T}_{3}$-E.A. in der Regel proportional (Abb. 4).

$\mathrm{Da}$ endogene Schilddrüsenhormone in Spiegeln, wie sie bei der Schilddrüsendiagnostik vorkommen, das Testergebnis kaum veränderten, kann somit der Testparameter als indirektes $\mathrm{Maß}$ für das gesamte spezifische Transportvermögen des Plasmas für Schilddrüsenhormone angesehen werden.

Diese Aussage trifft nicht zu:

1. Wenn der Spiegel endogener Schilddrüsenhormone das spezifische Transportvermögen des Plasmas übersteigt. Das Ergebnis wird dann stärker von Proteinfraktionen niedrigerer Affinität bestimmt. Solche Ergebnisse kommen möglicherweise bei Hyperthyreosen vor (Abb. 3).

2. Wenn bestimmte pathologische Plasmafaktoren vorliegen, die den. Testparameter unspezifisch beeinflussen. Ein Beispiel hierfür war die untersuchte Hyperlipämie (Abb. 4), bei der Serumlipide möglicherweise das Verteilungsgleichgewicht und die ${ }^{131} \mathrm{~J}-\mathrm{T}_{3}-\mathrm{An}-$ lagerung an Erythrocyten veränderten (21).

Abschließend sei auf eine Tatsache hingewiesen, die den Einfluß von Plasmaproteinen hoher Affinität zu Schilddrüsenhormonen auf das Testergebnis verstärkt.

Erythrocyten sind durch Adsorption von Plasmaproteinen im Blut wahrscheinlich von einer Hülle 
höherer Proteinkonzentration umgeben (22). Wurden Plasmaproteine hoher Affinität zu Schilddrüsenhormonen durch ${ }^{131} \mathrm{~J}-\mathrm{T}_{3}$ markiert, so lagerten sich bei einem Hämatokrit von 40 etwa $20 \%$ der Radioaktivität den Erythrocyten an und zwar sowohl bei euthyreoten Probanden als auch bei Graviden (s. Tab. $1 \%$ ${ }^{131} \mathrm{~J}-\mathrm{T}_{3}$-E.A. ungewaschener Erythrocyten). Die ${ }^{131} \mathrm{~J}-$ $T_{3}$-Verteilung $z$ wischen Proteinhülle und Erythrocyten wies bei niedrigen $T_{3}$-Konzentrationen . Unterschiede auf, indem bei Graviden der abwaschbare ${ }^{131} \mathrm{~J}-\mathrm{T}_{3}-\mathrm{An}$ teil deutlich vermehrt war (s. Tab. 1). Es kann daher angenommen werden, daß prozentual immer derselbe Anteil der im Plasma vorhandenen Proteine hoher $\mathrm{T}_{3^{-}}$ Affinität an die Erythrocyten angelagert wird. Quantitativ macht dieser bei erhöhtem Gehalt dieser Proteine im Plasma mehr, bei erniedrigtem Spiegel entsprechend weniger, als beim Normalfall aus.

Auf diese Weise wird, lediglič aufgrund der ProteinAdsorption das Gleichgewicht der Verteilung von ${ }^{131} \mathrm{~J}=\mathrm{T}_{3}$ bei Vermehrung des spezifischen Transportvermögens zugunsten der Hülle, bei dessen Verminderung zugunsten der Erythrocyten verschoben.

\section{Literatur}

1. Hamolsky, M. W., M. Stein und A. St. Freedberg, J. Clin. Endocr. Springfield 17,33 (1957). - 2. Mrtchell, M. L., A. B. HARDEN und M. E. O'RourKe, J. Clin. Endocr. Springfield 20,1474 (1960). - 3. Sterling, K. und M. Tabachirck, J. Clin. Endocr. Springfield 21, 456 (1961). - 4. Scriba, P. C., H. G. Hernze, R. Landgraf, K. W. Frey und K. Schwarz, Klin. Wschr. 44, 131 (1966). - 5. FreUNDLICH, H., Kapillarchemie Akad. Verlagsges., Leipzig (1930). - 6. Crispelt, K. R. und J. Coleman, J. Clin. Invest. 35, 475 (1956). - 7. FinK, G., Inaug. Diss. Freiburg (1962). 8. Ivgbar, S. H., Ann. N. Y. Acad. Sci. 86, 440 (1960). -9. PrtrRivers, R. und J. R. Tata, The thyroid hormones. Pergamon Press, London (1959). - 10. Robrins, J. und J. H. Nelson, J. Clin. Invest. 37, 153 (1958). - 11. Gordon, A. H., J. Gross, D. O'Connor und R. Pitt-Rivers, Nature, London 169, 19 (1952). 12. Horst, W. und H. Rösler, Klin. Wschr. 31, 13 (1953). -
13. Lakson, F., W. P. Dérss und E. C. Albright, Science Washington 115, 626 (1952). - 14. Derss, W. P., E. C. Albright und F. C. Larson, Proc. Soc. Exp. Biol. Med. (N. Y.) 84, 513 (1953). -15. Robins, J. und J. E. Rall, Recent Progr. Hormone Res. N. Y. 13, 161 (1957). - 16. Dowling, J. T., N. Freinkel und S. H. INGBAR, J. Clin. Endocr. Springfield 16, 280 (1956). - 17. RoBbins, J., J. E. Rall und M. L. Petermann, J. Clin. Invest. 36, 1333 (1957). - 18. ReCant, L., J. Clin. Invest. 35, 730 (1956). 19. Ureles, A. L. und M. Murray, J. Laborat. Clin. Med. S. Louị 54, 178 (1959). - 20. Walfish, P. G., A. Britton, R. Volpé und C. Ezrin, J. Clin. Endocr. Springfield 22, $178^{\circ}$ (1962). - 21. Meuret, G., Inaug. Diss. Freiburg (1963). - 22. Kreine, N. Ber. 9. Tag. dtsch. Ges. Bluttransf., Braunschweig 1960, Bibl. haemat. 12, 105 (1961). -
Dr. G. Meuret 78 Freiburg i. Br. Hugstetter Str. 55 